\title{
Trends in vitreoretinal surgery at a tertiary referral centre: 1987 to 1996
}

\author{
F G Ah-Fat, M C Sharma, M A Majid, J N McGalliard, D Wong
}

\begin{abstract}
Aim-To identify trends in vitreoretinal surgery at a tertiary referral centre from 1987 to 1996.

Methods-A retrospective study of patients who had undergone vitreoretinal surgery at St Paul's Eye Unit over two 6 month periods in 1987 and 1996. Preoperative ocular status, surgery details, and outcome were collected. $\chi^{2}$ and MannWhitney $U$ tests were used to analyse the data.

Results-The two periods under study were July to December 1987 and January to June 1996. 110 operations performed during 1987 (96 patients) and 330 operations during 1996 (289 patients) were analysed. There was a fourfold rise in the number of tertiary referrals and a sevenfold rise in the number of operations performed for conditions other than rhegmatogenous retinal detachment (RRD). Increasing indications for surgery included diabetic eye disease, macular hole, dropped nucleus, endophthalmitis, and subretinal neovascular membrane. There was a rise in the proportion of patients with RRD following cataract surgery (from $19.5 \%$ to $29.5 \%$ ). For both primary repair and reoperation, vitrectomy with internal tamponade was more commonly used in 1996. The anatomical success rate for primary repair changed from $76.6 \%$ to $84.7 \%$ after one operation and from $89.1 \%$ to $\mathbf{9 4 . 3 \%}$ following additional surgery.

Conclusions-This study points to a trend towards subspecialisation and tertiary referral in vitreoretinal surgery. Vitrectomy techniques are more commonly used for the primary repair of RRD and are applied to a wider spectrum of diseases. (Br F Ophthalmol 1999;83:396-398)
\end{abstract}

At the St Paul's Eye Unit in Liverpool, we have observed an increasing number of patients with rhegmatogenous retinal detachment (RRD)

Accepted for publication 22 September 1998

Table 1 Surgical activity fuly-December 1987 compared with fanuary-Fune 1996

\begin{tabular}{|c|c|c|}
\hline & 1987 & 1996 \\
\hline Total number of vitreoretinal procedures & 110 & 330 \\
\hline Rhegmatogenous retinal detachment (RRD) procedures* & 90 & 184 \\
\hline Number of patients ${ }^{\star}$ & 77 & 149 \\
\hline Internal referrals & 36 & 44 \\
\hline Tertiary referrals & 41 & 105 \\
\hline Procedures other than $\mathrm{RRD}^{\star}$ & 20 & 146 \\
\hline Number of patients ${ }^{\star}$ & 19 & 140 \\
\hline Internal referrals & 13 & 59 \\
\hline Tertiary referrals & 6 & 81 \\
\hline
\end{tabular}

${ }^{\star}$ Discrepancy due to some patients having more than one operation during the 6 month period. being referred for treatment since 1987. In addition, the indications for vitrectomy have widened over the past 10 years to include macular holes, ${ }^{1}$ epiretinal membranes, ${ }^{2}$ dropped nucleus, ${ }^{3}$ and subretinal neovascularisation. ${ }^{4}$ This trend towards subspecialisation and tertiary referral may be repeated in other units across the UK, with fewer general ophthalmologists performing RRD repair. These changes may have implications for the general training of ophthalmologists and for health service provision. The aim of this study is to document changes in the pattern of work in our unit in the past 10 years and to discuss its impact.

\section{Patients and methods}

Patients who had undergone vitreoretinal surgery at the St Paul's Eye Unit from July to December 1987 and from January to June 1996 were identified from the operating theatre register. The data set included details on the source of referral, previous surgery, lens status, extent of RRD, associated proliferative vitreoretinopathy (PVR), time interval between first attendance and surgery, and surgical technique. Outcome measures included the number of reoperations, anatomical success, and visual acuity. Anatomical success was defined as persisting retinal reattachment 6 months postoperatively. For patients who had been discharged, anatomical success and visual acuity were determined by contacting the referring hospital. Categorical data were analysed with the $\chi^{2}$ test and non-parametric continuous data with the Mann-Whitney U test.

\section{Results}

SURGICAL ACTIVITY

For the 6 month period in 1987, eight patients were lost to follow up and a further five patients had incomplete data sets. For 1996, seven patients were lost to follow up, while five had incomplete data sets. Available for analysis were 110 vitreoretinal operations on 96 patients during 1987, including 90 operations for RRD on 77 patients. For 1996, 330 operations on 289 patients, including 184 operations for RRD on 149 patients were analysed (Table 1). All surgery was performed by consultants and by fellows, senior registrars, and registrars training in the vitreoretinal unit. The main reasons for the threefold increase in surgical activity were an increase in the number of tertiary referrals and an increase in the number of operations performed for conditions other than RRD. In 1987, 36 patients undergoing surgery for RRD were internal referrals from within the Liverpool area compared with 44 for the corre- 
Table 2 Vitreoretinal procedures other than for rhegmatogenous retinal detachment during each 6 month period

\begin{tabular}{lcc}
\hline Procedure & 1987 & 1996 \\
\hline Vitrectomy for diabetic eye disease & 6 & 36 \\
Macular hole surgery & 0 & 25 \\
Vitreous biopsy/vitrectomy for & 2 & 11 \\
$\quad$ endophthalmitis & 0 & 9 \\
Vitrectomy for dropped nucleus & 1 & 8 \\
Removal of choroidal neovascular membrane & 1 & 5 \\
Peeling of epiretinal membrane & 0 & 2 \\
Removal of intraocular foreign body & 3 & 4 \\
Repair of posterior penetrating injury & 2 & 4 \\
Vitrectomy for branch retinal vein thrombosis & 2 & \\
Indirect laser (diabetic retinopathy, retinal & & 9 \\
$\quad$ break) & 0 & 3 \\
Drainage of choroidal haemorrhage & & \\
Vitrectomy/buckle for retinopathy of & & \\
$\quad$ prematurity & 0 & 2 \\
Miscellaneous & 5 & 28 \\
Total number of procedures $\dagger$ & 20 & 146 \\
Total number of patients $\dagger$ & 19 & 140 \\
\hline
\end{tabular}

*Includes removal of silicone oil, explant and dislocated intraocular implant, vitrectomy for malignant glaucoma, needlestick perforation, other types of vitreous haemorrhage, and prophylactic cryopexy.

†Discrepancy due to some patients having more than one operation during the 6 month period.

sponding period in 1996 . The number of tertiary referrals from other parts of the Mersey region for RRD had risen, however, from 41 patients in 1987 to 105 patients in 1996. In addition, large increases were noted in the number of patients undergoing treatment for conditions other than RRD. These included diabetic eye disease, macular hole, endophthalmitis, dropped nucleus, subretinal neovascular membrane, and epiretinal membrane (Table 2).

CHARACTERISTICS OF RHEGMATOGENOUS

RETINAL DETACHMENT

Ninety operations were performed on 77 patients for RRD in 1987. This had increased to 184 operations on 149 patients in 1996. In $1987,11.7 \%$ of patients had previously undergone primary RRD repair in another unit, compared with $5.4 \%$ in 1996 ( $\mathrm{p}=0.088$, $\chi^{2}$ test). There was a fall in the proportion of patients who were aphakic (from $14.3 \%$ to $4.7 \%$ ), but a rise in the proportion of patients who were pseudophakic (from $5.2 \%$ to $24.8 \%$ ) during the two periods under study. Overall, the proportion of patients with RRD who were aphakic or pseudophakic increased from $19.5 \%$ to $29.5 \%$ ( $p=0.103, \chi^{2}$ test). This was also accompanied by an increase in the proportion of patients who had significant PVR (grade B or worse) from $16.9 \%$ to $28.9 \%$ $\left(\mathrm{p}=0.048, \chi^{2}\right.$ test $)$ and a decrease in the proportion of RRD with macula still attached at initial presentation (from $49.4 \%$ to $38.3 \%$, $\mathrm{p}=0.109, \chi^{2}$ test). Along with the increase in surgical activity, the median time from first clinic attendance to surgery had risen from 5 days in 1987 to 16 days in 1996 (p 0.092 , Mann-Whitney U test).

SURGICAL APPROACH TO RHEGMATOGENOUS RETINAL DETACHMENT

Table 3 summarises the surgical approach for primary RRD repair for the two periods under study. In 1987, 64 patients underwent primary repair of RRD; $89.1 \%$ of patients were treated
Table 3 Surgical approach in primary rhegmatogenous retinal detachment repair

\begin{tabular}{lcc}
\hline & 1987 & 1996 \\
\hline Cryo and buckle/D-ACE & $57(89.1 \%)$ & $84(67.7 \%)$ \\
Vitrectomy +/- buckle/gas & $6(9.4 \%)$ & $24(19.4 \%)$ \\
Vitrectomy +/- buckle/silicone & $1(1.6 \%)$ & $16(12.9 \%)$ \\
$\quad$ oil & $64(100.0 \%)$ & $124(100.0 \%)$ \\
Total number of patients & \\
\hline
\end{tabular}

Table 4 Adjunctive measures in primary $R R D$ repair and reoperations

\begin{tabular}{lcc}
\hline & 1987 & 1996 \\
\hline Perfluorocarbon gas & $15(16.7 \%)$ & $38(20.7 \%)$ \\
Silicone oil & $6(6.7 \%)$ & $34(18.5 \%)$ \\
Perfluorocarbon liquid & $0(0.0 \%)$ & $31(16.9 \%)$ \\
Endolaser retinopexy & $3(3.3 \%)$ & $42(22.8 \%)$ \\
Retinectomy & $0(0.0 \%)$ & $11(6.0 \%)$ \\
Encirclement & $24(26.7 \%)$ & $12(6.5 \%)$ \\
Total number of procedures & $90(100.0 \%)$ & $184(100.0 \%)$ \\
\hline
\end{tabular}

by the conventional method with cryopexy and explant with or without drainage and air. In 1996, however, the proportion of primary RRD treated by the conventional method had decreased to $67.7 \%$ for 124 patients. Concurrently, there was a rise in the number of patients treated by vitrectomy (from $10.9 \%$ to $32.3 \%, p=0.0014, \chi^{2}$ test). Patients treated by an internal approach during primary surgery were often those with complicated detachments - for example, pseudophakic eyes with significant PVR. There were 26 reoperations for RRD in 1987 and 60 reoperations in 1996. During these operations, an internal approach with vitrectomy was commonly adopted $(57.7 \%$ in 1987 and $71.7 \%$ in 1996 , $\mathrm{p}=0.204, \chi^{2}$ test). In addition, from 1987 to 1996, we recorded an overall increase in adjunctive measures such as retinectomy, the use of perfluorocarbon liquid, endolaser retinopexy, and internal tamponade and a decrease in the number of encirclements (Table 4). The mean number of reoperations after initial failure, including those beyond the study periods, was 1.3 (range 1-3) for the 1987 cohort, compared with 2.2 for the 1996 cohort (range $1-5)$.

ANATOMICAL AND FUNCTIONAL SUCCESS

The median interval between surgical repair and the last recorded outpatient visit was 16.2 months for patients in the 1987 group and 12.3 months for those in the 1996 group ( $p$ 0.166, Mann-Whitney U test). In 1987, 76.6\% of patients who had undergone primary RRD repair had persisting retinal reattachment 6 months after one operation. After additional surgery, the anatomical success rate for 1987 was $89.1 \%$. In 1996 , the anatomical success rates for primary RRD repair were $84.7 \%$ after one operation and $94.3 \%$ following additional surgery. These increases in anatomical success did not however reach statistical significance ( $p=0.171$ and 0.190 respectively, $\chi^{2}$ test).

While anatomical results had improved in 1996 (though not to a statistically significant degree), differences in the visual results of the two cohorts were less evident. $45.3 \%$ of patients undergoing primary RRD repair achieved a visual acuity of $6 / 18$ or better in 
1987, compared with $46.8 \%$ in 1996 $\left(p=0.849, \chi^{2}\right.$ test $)$. We believe these findings may be partly explained by the longer time interval from diagnosis to surgery and the increases in the incidence of macular detachment in the 1996 cohort.

OTHER PROCEDURES

Significant rises were noted in the number of operations performed for conditions other than RRD (Table 2). During the 6 month period under study in 1987, we performed six vitrectomy procedures for the complications of diabetic retinopathy. In the corresponding period in 1996, the number of vitrectomies had risen to 36 , the increase being accounted by both internal and tertiary referrals. We feel that these increases were likely to be due to a trend towards earlier referral and treatment rather than to changes in the pattern of the disease. In recent years, vitrectomy with intraocular gas tamponade has been increasingly applied for the treatment of macular holes and accounted 25 surgical procedures in our unit during the period under study in 1996. We also performed eight operations for the treatment of subretinal neovascular membrane and five operations for epiretinal membrane during that period. Increases were similarly recorded in the number of vitrectomies performed for dropped nucleus after phacoemulsification surgery (nine patients in 1996) and vitrectomies or vitreous biopsies for endophthalmitis (from two to 11).

\section{Discussion}

This study points to a trend towards subspecialisation and tertiary referral in vitreoretinal surgery. We perceive a change in professional attitude with respect to RRD repair in recent years. A decade ago, the treatment of RRD in the Mersey region was mainly the responsibility of the general ophthalmologist. Recent advances meanwhile have led to a wider use of expensive technology in vitreoretinal surgery, including indirect operating systems with wide angled optics, perfluorocarbon liquids, and retinectomy. Coupled with the growth of day case surgery and its implications on the provision of beds and theatre resources, surgeons working in general ophthalmic units may now be less well equipped in treating RRD. In a 1995 survey, we found that most general ophthalmologists in this region had abandoned RRD repair, preferring to refer their patients to a specialised unit. ${ }^{5}$ This is borne out by the findings of the current study which shows that in addition to increases in surgical activity, only $5.4 \%$ of our patients had undergone primary RRD repair in another unit in 1996, compared with $11.7 \%$ in 1987 . With a wider range of surgical techniques available to treat patients at the first operation, including vitrectomy with internal tamponade, subspecialisation must be seen as a positive step. In our unit, we have recorded an increase in the success rate for primary surgery, though this did not reach statistical significance.

Subspecialisation has a number of implications for ophthalmologists in training. Exposure to the subspecialty may be reduced as training is delivered by consultants who do not practise vitreoretinal surgery. Ophthalmologists who do not intend to practise as vitreoretinal surgeons may no longer need to receive hands on training in RRD repair. However, it is desirable for ophthalmologists in training to spend a period of time in a vitreoretinal unit where they can gain experience in the diagnosis and assessment of RRD and appreciate the spectrum conditions that can be treated by the vitreoretinal surgeon.

The move towards subpecialisation and tertiary referrals must not act as a barrier to fast and efficient care. Our study reveals that the increase in tertiary referral has been accompanied by a rise in the interval from first attendance to surgery and a fall in the percentage of cases of RRD operated on before the macula detached. It is therefore important that vitreoretinal centres should have adequate resources to avoid delays in assessment and treatment. In our unit, we have found that delays have been minimised by the appointment of a vitreoretinal nurse practitioner to whom referrals are made and by the provision of open access clinics for referring ophthalmologists and patients. To provide a continuous and responsive service, it is equally important to maintain an adequate staffing level to cover absences of individual surgeons for leave, $\mathrm{CME}$, teaching or research. Finally, it is essential for vitreoretinal units to perform regular audits and to implement measures aimed at improving both the delivery and the quality of care.$^{6}$ In that context, we anticipate that the current study will form the basis for future audits in this unit.

1 Kelly NE, Wendel RT. Vitreous surgery for idiopathic macular holes: results of a pilot study. Arch Ophthalmol 1991;109:654-9.

2 de Bustros S, Thompson JT, Michels RG, et al. Vitrectomy for idiopathic epiretinal membranes causing macular for idiopathic epiretinal membranes

3 Gilliland GD, Hutton WL, Fuller DG. Retained intravitreal Gilliland GD, Hutton WL, Fuller DG. Retained intravitreal
lens fragments after cataract surgery. Ophthalmology 1992;99:1263-9.

4 Berger AS, Kaplan HJ. Clinical experience with the surgical removal of subfoveal neovascular membranes: short-term postoperative results. Ophthalmology 1992;99:969-76.

5 Wong D, McGalliard J. Are we getting better at treating retinal detachment? Technology, referral pattern or primary care? Eye 1997;11:763-4

6 Sullivan PM, Luff AJ, Aylward GW. Results of primary retinal reattachment surgery: a prospective audit. Eye 1997;11: 869-71. 